\title{
Literacia e inclusão digital: Um estudo piloto com jovens em vulnerabilidade social na cidade de Natal-RN
}

Literacy and digital inclusion: A pillot study with young people in social vulnerability in the city of Natal-RN

Alfabetización e inclusión digital: Un estudio piloto sobre la vulnerabilidad social de los jóvenes en la ciudad de Natal-RN

\section{Resumo}

Este trabalho apresenta um recorte de uma investigação que teve por objetivo analisar a inclusão digital de jovens moradores de Natal no Estado do Rio Grande do Norte (RN). Trata-se de uma pesquisa piloto, quantitativa, exploratória e descritiva na qual participaram 21 jovens entre 15 e 18 anos de idade. Os dados foram organizados com o apoio de estatística descritiva e inferencial. Os softwares SPSS, Minitab e R foram utilizados para calcular médias, desvio-padrão e realizar a Análise de Cluster. Os resultados revelam que os jovens possuem acesso à Internet em seus domicílios, a partir de conexão wi-fi e, principalmente, rede móvel. O smartphone se revelou como o principal dispositivo de acesso (90\%). Cerca de 66\% utilizam a Internet para acessar redes sociais e aplicativos de mensagens e $47 \%$ para estudar. Quanto à literacia digital, os resultados mostram médias satisfatórias nas perguntas relacionadas à programação de computadores, no entanto, revelam algumas dificuldades relacionadas à (1) edição e à criação de conteúdos, (2) segurança e comunicação e (3) habilidades de pesquisa. Esta pesquisa oferece indicativos para o debate acerca da inclusão digital de jovens em vulnerabilidade, moradores de comunidades periféricas do supracitado município.

Palavras-chave: Literacia digital; Inclusão digital; Jovens em vulnerabilidade social.

\begin{abstract}
This paper presents a part of an investigation which aims to analyze the digital inclusion of young residents of Natal, a city in the State of Rio Grande do Norte (RN), Brazil. A quantitative, exploratory and descriptive pilot research was conducted with twenty-one young people, aged between 15 and 18 years old. The data were organized with the support of descriptive and inferential statistics. SPSS, Minitab and R software were used to calculate averages, standard deviations and perform Cluster Analysis. The results show that they have access to the Internet at home, through wi-fi and, principally, mobile network connections. The smartphone has revealed itself as the main access device (90\%). About $66 \%$ use the Internet to access social networks and messaging applications and $47 \%$ to study. As
\end{abstract}


for digital literacy, the results show satisfactory averages related to computer programming. However, they also show some difficulties related to (1) editing and content creation, (2) security and communication, (3) and research skills. This research offers indications for the debate about the digital inclusion of young people in vulnerability, living in peripheral communities of the aforementioned municipality.

Keywords: Digital literacy; Digital inclusion; Youth at risk.

\section{Resumen}

Este trabajo presenta un recorte de una investigación que tuvo por objetivo analizar la inclusión digital de jóvenes habitantes de Natal en el Estado Rio Grande do Norte (RN). Se trata de un estudio piloto, cuantitativo, exploratorio y descriptivo en el que participaron 21 jóvenes entre 15 y 18 años de edad. Los datos se organizaron con el apoyo de estadísticas descriptivas e inferenciales. Se utilizó los softwares SPSS, Minitab y R para calcular medias, desviación estándar y realizar el Análisis de Clúster. Los resultados revelan que los jóvenes poseen acceso a Internet en sus domicilios, a partir de conexión wi-fi y, principalmente, por red móvil. El teléfono inteligente se ha revelado como el principal dispositivo de acceso (90\%). Alrededor del 66\% utiliza Internet para acceder a redes sociales y aplicaciones de mensajería y $47 \%$ para estudiar. En cuanto a la alfabetización digital, los resultados muestran medias satisfactorias en las preguntas relacionadas con la programación de computadoras, sin embargo, revelan algunas dificultades relacionadas con la (1) edición y la creación de contenidos, (2) seguridad y comunicación y (3) habilidades de investigación. Este estudio ofrece indicativos para el debate acerca de la inclusión digital de jóvenes en vulnerabilidad, habitantes de comunidades periféricas del citado municipio.

Palabras clave: Alfabetización digital; Inclusión digital; Jóvenes en vulnerabilidad social.

\section{Introdução}

Este artigo apresenta um recorte de uma pesquisa de doutorado, a qual analisa a resiliência e a inclusão digital entre jovens brasileiros que estudam Tecnologia da Informação (TI) e tem como objetivo principal investigar se uma maior literacia digital proporciona um incremento na resiliência de jovens em vulnerabilidade social. Para esse recorte, foi considerado como objetivo geral analisar a inclusão digital de jovens em situação de vulnerabilidade da zona oeste da cidade de Natal no Estado do Rio Grande do Norte (RN), Brasil. Como objetivos específicos, pode-se citar: a) Realizar um levantamento do perfil dos jovens no que diz respeito à situação socioeconômica e à inclusão digital e; b) Investigar os níveis de literacia digital de jovens de periferia.

O debate da inclusão digital nos levou a esta investigação quantitativa, tendo em vista o quanto este campo de estudos tem se tornado importante para pensar a questão da educação nos dias atuais, em particular no Brasil, onde vem sendo bastante problematizada atendendo ao cenário de: (a) acesso à Internet; (b) acesso a equipamentos e; (c) literacia (letramento) digital. Cada uma das variáveis que compõem este tripé merece ponderações, para consolidar o uso das tecnologias digitais como elementos de mediação nos processos de ensino e aprendizagem, inserção cidadã na sociedade e nas políticas educacionais do País.

\section{Enquadramento Teórico}

No Brasil, a política pública de inclusão digital está voltada para temáticas como cidadania, inclusão social, desenvolvimento local e nacional (Costa et al., 2019; Rodrigues \& Batista, 2018). Todavia, Silva et al. ressaltam que mesmo com a expansão tecnológica oportunizando acesso ao computador, considerando sua variedade de dispositivos (desktop, notebook, tablet e smartphone) e à Internet, “(...) sabe-se que ainda há uma grande quantidade de pessoas que não se encontram preparadas para o manuseio de tais ferramentas, sendo excluídas digitalmente do contexto social” (Silva et al., 2019, p. 228). Conforme Robinson (2018), conectividade constante é a condição sine qua non da inclusão social. Para Sorj (2003, p. 59), a exclusão digital possui intensa conexão com as outras formas de desigualdade social e, comumente, as taxas mais altas de exclusão digital encontram-se nas camadas populacionais de renda mais baixa. De acordo com Hamelink (2001), as tecnologias digitais podem ser consideradas um bem essencial para a igualdade social e para o desenvolvimento humano tal como a água, a energia ou o sistema rodoviário. Apesar de não hipotecarem a sobrevivência humana, são um fator fundamental 
de inclusão social.

Segundo Robin (2008), literacia digital é a capacidade de comunicar, discutir, reunir informações e ser capaz de pedir ajuda no mundo digital. Para Palmeiro, Pereda e Aires (2017, p. 98) "[...] investigações realizadas, a fim de avaliar o impacto dos chamados programas de literacia digital têm sido desenvolvidas, principalmente, a partir de uma perspectiva tecnológica e simplista". Todavia, o conceito de literacia digital adotado neste trabalho compreende a aprendizagem da tecnologia digital para além da simples instrumentalização e como via de inserção cidadã na sociedade. Desse modo, diz respeito ao uso social amplo das tecnologias digitais pelo indivíduo, para o exercício pleno de sua cidadania no mundo hodierno. A literacia digital é, desta feita, considerada necessária para fornecer desenvolvimento sustentável (Radovanović et al., 2020), empregabilidade, acesso a bens e serviços e é crucial para superar o fosso digital.

A sociedade atual vive imersa em um mundo digital, o que requer habilidades, conhecimentos e atitudes dos cidadãos para acompanhar o processo de evolução. Lyotard (1988), filósofo francês, há muito referia que é preciso que o cidadão se adapte à evolução tecnológica para acompanhar o processo de mudança que ocorre no mundo. Trata-se de um processo natural de mudanças, no qual as tecnologias têm um destaque pelas possibilidades que oferecem à sociedade, nas diversas esferas, seja social, profissional, educacional.

Moran (2018a) considera que a era digital insere os cidadãos em um novo cenário, onde o mundo virtual e o físico se complementam, se interconectam de modo a se tornarem praticamente inseparáveis. A conexão com o mundo digital possibilita um novo tipo de comunicação, novas formas de aprendizagem, de compras, pagamentos, e outros serviços, o que reflete em um novo modo de vida. Deste modo, o referido autor ainda salienta que a falta de acesso exclui o cidadão dos aspectos fundamentais para a vida profissional, a interação com os outros e para com os diversos tipos de serviços.

No âmbito educacional, a conexão com as redes digitais permite acesso a ampla informação, proporcionando pesquisas em variadas plataformas, debates, publicações online, e de uma variedade de outros serviços (Moran, 2018a). As tecnologias digitais podem proporcionar um importante contributo à educação de modo amplo, desde o apoio a modalidades de ensino, sendo estas presencial, a distância ou on-line, e a semipresencial, à utilização de vários recursos digitais para o ensino e aprendizagem, avaliação. São várias as possibilidades de uso, em qualquer tempo e lugar, através de smartphone, tablet, desktop ou notebook, utilizando, editando ou criando diversos tipos de atividades, inserindo os alunos como protagonistas da construção do conhecimento (Moran, 2018b, 2018a).

As tecnologias digitais integradas aos dispositivos móveis, tendo em conta a mobilidade, poderão facilitar a organização da vida diária em qualquer local, seja para atividades pessoais, sociais, educacionais e de trabalho. Através de acesso por meio de aplicativos, é possível aceder a diferentes formas de comunicação: acesso à localização; estabelecimentos, públicos ou privados, tais como bancos, lojas, aeroporto, rodoviária, estacionamentos, etc.; participar de reuniões; receber, digitar e compartilhar documentos; elaborar material diversos para diversos fins, etc. (Araujo \& Vilaça, 2016). Percebe-se que os dispositivos móveis com acesso às tecnologias digitais passam a ter um papel essencial no cotidiano, o que reflete a necessidade de todos os cidadãos estarem conectados.

Atualmente, a Internet das coisas vem invadindo os espaços físicos conectando objetos à Internet, de modo a transformar informações em serviços, atendendo necessidades de um dispositivo ou do cidadão. Isso representa um benefício para o cidadão no seu cotidiano, para as empresas, o que pode ser considerado um "um novo ciclo de inovação, tanto tecnológico como de processos e negócios sociais" (Albertin \& Albertin, 2017, p. 15). Essa evolução já começa a refletir na vida cotidiana o que implica em uma atenção para oportunidades de conhecimento pois a tendência é uma mudança no âmbito socioeconômico.

Neste cenário, é importante mencionar algumas referências de medidas da literacia digital que abordam aspectos relevantes na formação dos cidadãos, as quais foram utilizadas como base para este trabalho: DigComp2.1 (Lucas \& Moreira, 
2017); o Technology Readiness Index - TRI 2.0 (Parasuraman \& Colby, 2015) e o Development and validation of the Internet Skills Scale - ISS (Van Deursen, Helsper \& Eynon, 2016). A DigComp2.1 (Lucas \& Moreira, 2017) está estruturada num quadro de referência Europeu de classificação do nível de literacia digital dos cidadãos. Compreende 5 áreas de competência, as quais estão relacionadas com: literacia e informação; comunicação e colaboração; criação de conteúdo digital; segurança e; resolução de problemas. Este documento descreve atividades em cada uma das áreas de competência descritas, para reflexão relacionada às práticas utilizadas.

O TRI 2.0 (Parasuraman \& Colby, 2015) corresponde a uma escala de 16 itens que mede a prática dos cidadãos em relação às tecnologias. Esta escala corresponde a itens relacionados ao otimismo em relação às tecnologias e também a aspectos relacionados à inovação, desconforto e insegurança.

Já ISS (Van Deursen et al., 2016) é um instrumento elaborado para medir as habilidades na Internet. Consiste em cinco tipos de habilidades relacionadas à Internet: operacional, informação de navegação, social, criativo e móvel.

\section{Metodologia}

Esta pesquisa é de cunho quantitativo e tem foco em um recorte de um estudo piloto realizado em dezembro de 2019 com jovens ligados a uma Organização Não-Governamental (ONG) localizada na zona oeste da cidade de Natal (RN). Classifica-se também como pesquisa exploratória (Gil, 2009) e descritiva (Gonçalves, 2005). Participaram da amostra 21 jovens entre 15 e 18 anos de idade que tiveram autorização de seus pais, os quais assinaram o Termo de Consentimento Livre e Esclarecido (TCLE).

A coleta de dados ocorreu por meio de questionário impresso com questões fechadas. O instrumento original foi composto por 88 itens, na sua maioria utilizando a escala Likert de 5 pontos. Para esse recorte, foram analisados 40 itens, constituído por questionário sociodemográfico para compreensão do contexto do estudante e suas redes de apoio e questões acerca de inclusão e literacia digital. Parte das perguntas do instrumento de inclusão digital teve como base Lucas e Moreira (2017), Parasuraman e Colby (2015) e Van Deursen et al. (2016).

Para a organização e a análise dos dados, recorreu-se à estatística descritiva e inferencial. Utilizaram-se os softwares SPSS, Minitab e R, em que foi possível calcular médias, desvio-padrão e realizar a análise de cluster. A análise de agrupamento (cluster) foi utilizada com intuito de agrupar os jovens com características comuns quanto às variáveis do instrumento de literacia digital composto de 22 itens medidos por uma escala de Likert. A distância euclidiana foi utilizada para verificar a proximidade entre os elementos. Assim, foram construídos dois grupos (1 e 2) como apresentado na Figura 1.

\section{Resultados e Discussão}

\subsection{Perfil Sociodemográfico}

No que diz respeito às variáveis sociodemográficas (Tabela 1), dos 21 respondentes, 80,95\% ( $\mathrm{n}=17)$ declaram-se homem cis, 19,05\% $(\mathrm{n}=4)$ mulher cis, destacando desproporção com a realidade nacional. Sobre a raça ou cor, 57,14\% $(\mathrm{n}=12)$ revelaram ser pardos e 42,86\% (n=9) se autodeclararam como negros; desse modo, todos caracterizados como integrantes de grupo foco de políticas afirmativas brasileiras. Já 38,10\% (n=8) dos respondentes declararam que não possuem nenhuma religião, seguidos de 33,33\% (n=7) católicos e 28,57\% (n=6) evangélicos, que são as religiões mais representativas no Brasil, conforme apontam os dados do Instituto Brasileiro de Geografia e Estatística (IBGE, 2013). 
Tabela 1: Variáveis Sociodemográficas: Moradia e Renda Familiar.

\begin{tabular}{c|c|c}
\hline Variáveis & $\mathbf{N}$ & $\mathbf{\%}$ \\
\hline Renda Familiar & & \\
Atté 1 salário mínimo & & 52,38 \\
De 1 a 2 salários mínimos & 08 & 38,10 \\
De 2 a 4 salários mínimos & 01 & 04,76 \\
De 4 a 10 salários mínimos & 01 & 04,76 \\
De 10 a 20 salários mínimos & 00 & 00,00 \\
Mais de 20 salários mínimo & 00 & 00,00 \\
\hline Total & $\mathbf{2 1}$ & $\mathbf{1 0 0 , 0 0}$ \\
\hline Com quem reside & & \\
Esposo & 01 & 04,76 \\
Mãe & 02 & 09,52 \\
Mãe e avó & 01 & 04,76 \\
Mãe e primos & 04,76 \\
Pais & 07 & 33,33 \\
Pais e irmãos & 33,33 \\
Tia & $\mathbf{2 1}$ & $\mathbf{1 0 0 , 0 0}$ \\
\hline Total & 04,76 \\
\hline
\end{tabular}

n=frequência absoluta. Fonte: Autores.

No aspecto moradia, 33,33\% $(n=7)$ vivem com os pais e irmãos, $71,43 \%(n=15)$ desses estudantes, sempre viveram com essas pessoas; cerca de $28 \%(\mathrm{n}=06)$ consideram os pais biológicos e irmãos como sua família. Conforme a Tabela 1 aponta, 52,38\% (n=11) compartilham menos de um salário mínimo com suas famílias e 38,10\% (n=08) até dois salários mínimos (cerca de 2 mil reais). Esses valores comprometem bastante a qualidade de vida dessas famílias considerando que o custo médio para se viver na cidade era de R \$ 4.649,03 no biênio 2017-2018, conforme aponta o IBGE (2019). É importante notar que a exclusão digital é mais evidente para pessoas que não conseguem acessar ou comprar tecnologia em razão de variáveis sociodemográficas como sexo, status socioeconômico, moradia em zona urbana ou rural, assim como níveis de alfabetização (Hargittai \& Hinnant, 2008; Radovanović et al., 2020).

\subsection{Inclusão Digital}

\subsubsection{Acesso e uso da Internet e dos equipamentos}

A Tabela 2 apresenta as respostas dos estudantes referentes ao instrumento de inclusão digital. Percebeu-se que 90,48\% ( $\mathrm{n}=19)$ dos estudantes utilizam mais a Internet em casa, seguidos de em casa de vizinhos, parentes ou amigos $(33,33 \%)$ e locais públicos com rede aberta $(28,57 \%)$. Outro aspecto mencionado refere-se à finalidade que costuma acessar à Internet, sendo que 66,67\% (n=14) utilizam para acessar redes sociais ou aplicativos de mensagens, seguidos de uso para lazer e jogar com 61,90\% $(n=13)$ e 47,62\% $(n=10)$ utilizam para estudar ou realizar trabalhos escolares. Para o tipo de Internet que possui, $61,90 \%(\mathrm{n}=13)$ possuem conexão wi-fi por meio de provedor de Internet e o serviço de Internet móvel (3G/4G) aparece com $38,10 \%$ (n=08). Percebeu-se ainda que o smartphone é o principal dispositivo que possuem com 90,48\% (n=19). Apenas 5 jovens afirmaram possuir notebook. 
Tabela 2: Acesso à Internet e a equipamentos.

\begin{tabular}{|c|c|c|}
\hline Onde costuma acessar à Internet* & $\mathrm{N}$ & $\%$ \\
\hline Em casa & 19 & 90,48 \\
\hline Em casa de vizinhos, parentes ou amigos & 07 & 33,33 \\
\hline No local em que estuda & 03 & 14,29 \\
\hline No trabalho & - & - \\
\hline Em Lan house & 03 & 14,29 \\
\hline Através do celular & 06 & 28,57 \\
\hline Em locais públicos com rede aberta & 03 & 14,29 \\
\hline Não costuma acessar à Internet & - & - \\
\hline \multicolumn{3}{|l|}{ 2. Finalidade você costuma acessar à Internet* } \\
\hline Para ler notícias e atualidades & 09 & 42,86 \\
\hline Para lazer e/ou jogar & 13 & 61,90 \\
\hline Para acessar redes sociais ou aplicativos de mensagens & 14 & 66,67 \\
\hline Para trabalhar, ou seja, em contextos profissionais & 01 & 4,76 \\
\hline Para estudar ou realizar trabalhos escolares & 10 & 47,62 \\
\hline Para realizar compras & 01 & 4,76 \\
\hline Para buscar oportunidade de emprego & 06 & 28,57 \\
\hline Para outras finalidades & 04 & 19,05 \\
\hline \multicolumn{3}{|l|}{ 3. Tipo de Internet que possui na sua casa* } \\
\hline Não possuo acesso à Internet em minha residência & 01 & 4,76 \\
\hline $3 \mathrm{G} / 4 \mathrm{G}$ & 08 & 38,10 \\
\hline Conexão wi-fi através de provedor de Internet da minha cidade & 13 & 61,90 \\
\hline Internet compartilhada do vizinho ou familiares & 02 & 9,52 \\
\hline \multicolumn{3}{|l|}{ 4. Tipo de equipamento que possui* } \\
\hline Celular do tipo Smartphone & 19 & 90,48 \\
\hline Computador & 03 & 14,29 \\
\hline Notebook & 05 & 23,81 \\
\hline Tablet & - & - \\
\hline Não possuo nenhum dos equipamentos listados & 01 & 4,76 \\
\hline
\end{tabular}

*Foram contabilizados o "sim" de cada resposta, pois trata-se uma pergunta de múltipla escolha com possibilidade de marcar mais de uma alternativa. Portanto, o somatório ultrapassa 21 respostas. Fonte: Autores.

A questão do acesso à Internet merece atenção e reflexão. Geralmente se pensa que cidadãos de classes mais desfavorecidas não têm condição de se alimentar, menos ainda de ter Internet em casa. Os dados de nosso estudo revelam que 90\% dos jovens acessam à Internet em casa, um índice maior que o apontado pelo Instituto Brasileiro de Geografia e Estatística (IBGE, 2018), o qual afirma que a rede chega em 8 a cada 10 domicílios brasileiros. O acesso via lan house é inexpressivo (como se vê na Tabela 2), o que é interessante porque, há uma década, este era um dos locais mais populares nos bairros de periferia para acesso às redes sociais, jogos ou execução de trabalhos escolares. $\mathrm{O}$ acesso no local em que estuda e locais públicos também tem pouca relevância, conforme as respostas obtidas.

O acesso pelo celular é uma informação relevante: primeiro porque na questão 01, o dispositivo móvel aparece com apenas $28 \%$ das respostas, o que faz gerar dúvida, se os $90 \%$ que acessam em casa fazem por meio de outros dispositivos. No entanto, a questão 04 indica que $90 \%$ do equipamento para acesso à Internet é mesmo o celular, e que é mais fácil ter notebook do que computador do tipo desktop, o que destaca uma predileção para dispositivos móveis. É importante observar que 99,2\% dos brasileiros utilizam Internet, o fazem por meio do celular, já 48,1\% acessam a partir de computador, $23,3 \%$ por meio da TV e apenas 13,4\% afirmaram usar tablet, de acordo com o IBGE (2018).

Pode-se afirmar que por mais desfavorecido economicamente que seja, é sempre possível adquirir um aparelho de smartphone e acessar Internet, seja ela gratuita, paga ou compartilhada. Em uma pesquisa, Van Deursen (2013) descobriu que pessoas com baixos níveis de escolarização e pessoas com deficiência estão usando a Internet por mais horas por dia em seu tempo livre, do que as populações com formação em nível superior e empregadas. Pela nossa experiência em espaços periféricos, a quase totalidade dos jovens usa smartphones para jogar, acessar redes sociais ou fazer tarefas escolares: uma 
questão que se torna cada vez mais importante, considerando modelos de trabalho remoto e híbrido no horizonte da educação brasileira. Uma pesquisa intitulada TIC Kids Online Brasil, com crianças e jovens entre 9 e 17 anos, constatou que mais de $90 \%$ deles utilizam smartphone para acessar a rede. Conforme esse estudo, verificou-se um aumento no acesso à Internet para assistir "vídeos, programas, filmes ou séries (83\%), ouvir música on-line (82\%). Atividades multimídia passaram a ser as mais realizadas entre as crianças e os adolescentes usuários de Internet no Brasil, superando pesquisas na Internet para trabalhos escolares (74\%)" (Comitê Gestor de Internet no Brasil, 2019, p. 109). É, portanto, fundamental discutir a qualidade desse acesso em todos os níveis de ensino e franquia de dados que essa população tem disponível.

No que diz respeito à experiência com cursos na modalidade de Educação a Distância (EaD), que é a modalidade de educação que mais cresce no país, 38,10\% dos respondentes declararam não ter feito nenhum curso, enquanto que a maioria informou já ter passado pela experiência com EaD como mostra a Tabela 3.

Tabela 3: Experiência com EaD.

\begin{tabular}{l|l|l}
\hline Faz ou fez algum curso a distância & $\mathrm{N}$ & $\%$ \\
\hline Não & 08 & 38,10 \\
Sim, pelo menos um & 08 & 38,10 \\
Sim, mais de um curso & 04 & 19,05 \\
Não respondeu & 01 & 4,76 \\
\hline Total & $\mathbf{2 1}$ & $\mathbf{1 0 0 , 0 0}$ \\
\hline
\end{tabular}

n=frequência absoluta. Fonte: Autores.

\subsubsection{Literacia Digital}

A Tabela 4 apresenta o comportamento médio da análise de agrupamento (cluster). O desvio-padrão (DP) abaixo de 1, na maioria dos itens, revela uma amostra mais homogênea. 
Tabela 4: Literacia digital segundo análise agrupamento.

\begin{tabular}{|c|c|c|c|c|c|c|c|}
\hline \multirow{2}{*}{ Área } & \multirow{2}{*}{ Instrumento de literacia digital } & \multicolumn{3}{|c|}{ Grupo 1} & \multicolumn{3}{|c|}{ Grupo 2} \\
\hline & & $\mathrm{n}$ & $\mathrm{Me}$ & DP & $\mathrm{n}$ & $\mathrm{Me}$ & DP \\
\hline \multirow{5}{*}{ 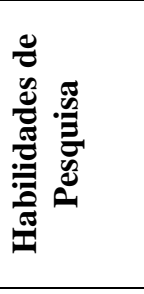 } & $\begin{array}{l}\text { Acho fácil decidir quais são as melhores palavras-chave a } \\
\text { utilizar quando faço pesquisas online }\end{array}$ & 16 & 3,00 & 0,73 & 05 & 4,00 & 1,23 \\
\hline & Acho fácil encontrar um site que visitei antes & 16 & 2,67 & 0,87 & 05 & 4,00 & 0,71 \\
\hline & $\begin{array}{l}\text { Acho clara a maneira como muitos sites são desenhados e } \\
\text { apresentados visualmente }\end{array}$ & 16 & 3,13 & 0,96 & 05 & 3,60 & 0,55 \\
\hline & Sinto que tenho facilidade de encontrar informações online & 15 & 2,93 & 1,16 & 05 & 3,80 & 1,64 \\
\hline & Sei verificar a credibilidade de conteúdos digitais & 16 & 2,93 & 0,68 & 05 & 4,00 & 1,73 \\
\hline \multirow{5}{*}{ 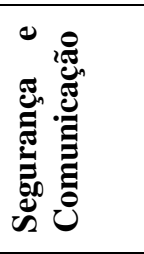 } & ações devo e não devo compartilhar & 16 & 3,87 & 0,89 & 05 & 4,60 & 0,55 \\
\hline & $\begin{array}{l}\text { Tenho cuidado de tomar meus comentários e comportamentos } \\
\text { adequados à situação em que me encontro online }\end{array}$ & 16 & 3,63 & 0,89 & 05 & 4,80 & 0,45 \\
\hline & Sei como alterar com quem compartilho conteúdo online & 16 & 4,00 & 0,97 & 05 & 4,40 & 0,89 \\
\hline & Sei como ajustar as minhas configurações de pri & 16 & 3,94 & 0,85 & 05 & 4,40 & 0,55 \\
\hline & são seguros para baixar & 16 & 3,50 & 1,03 & 05 & 3,80 & 1,30 \\
\hline \multirow{5}{*}{ 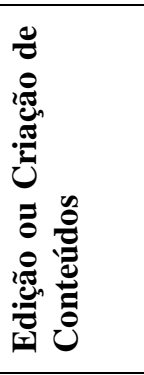 } & $\begin{array}{l}\text { Sei como criar algo novo a partir de imagens online } \\
\text { existentes, música ou vídeo }\end{array}$ & 16 & 3,44 & 0,73 & 05 & 4,40 & 0,55 \\
\hline & $\begin{array}{l}\text { Sei como fazer mudanças básicas em conteúdo que outras } \\
\text { pessoas produzem }\end{array}$ & 16 & 3,06 & 0,57 & 05 & 3,40 & 1,14 \\
\hline & Sei como criar um site & 16 & 2,38 & 0,81 & 05 & 4,40 & 0,89 \\
\hline & $\begin{array}{l}\text { Sei quais são os tipos diferentes de licenças que se aplicam ao } \\
\text { conteúdo online }\end{array}$ & 16 & 3,19 & 0,75 & 05 & 4,20 & 0,84 \\
\hline & $\begin{array}{l}\begin{array}{l}\text { Sei como utilizar ferramentas digitais para trabalhos } \\
\text { colaborativos }\end{array} \\
\end{array}$ & 15 & 3,73 & 1,22 & 04 & 3,75 & 1,50 \\
\hline \multirow{3}{*}{ 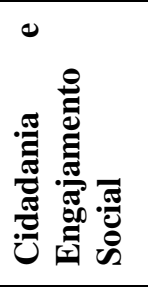 } & $\begin{array}{l}\text { Sei fazer uso de ferramentas digitais para mobilizar pessoas a } \\
\text { lutarem por uma causa, se empoderarem e exercerem a } \\
\text { cidadania }\end{array}$ & 15 & 3,20 & 0,94 & 05 & 3,40 & 0,89 \\
\hline & $\begin{array}{l}\text { Eu me sinto seguro em selecionar a indicar tecnologias } \\
\text { digitais assistivas }\end{array}$ & 15 & 3,20 & 0,68 & 05 & 3,20 & 0,84 \\
\hline & $\begin{array}{l}\text { Sou capaz de reconhecer os impactos ambientais decorrentes } \\
\text { do uso e da criação de tecnologias digitais }\end{array}$ & 15 & 3,27 & 0,80 & 05 & 4,00 & 0,71 \\
\hline \multirow{4}{*}{ 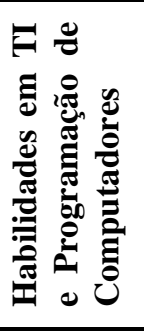 } & $\begin{array}{l}\text { Estou seguro de que sistemas tecnológicos farão o que eu } \\
\text { programar para eles fazer }\end{array}$ & 15 & 3,53 & 1,13 & 05 & 4,40 & 0,89 \\
\hline & $\begin{array}{l}\text { Gosto do desafio de compreender equipamentos de alta } \\
\text { tecnologia }\end{array}$ & 14 & 3,71 & 0,83 & 05 & 4,20 & 1,30 \\
\hline & $\begin{array}{l}\text { Sinto que tenho menos problemas que as outras pessoas para } \\
\text { fazer a tecnologia trabalhar para mim }\end{array}$ & 14 & 3,57 & 0,85 & 05 & 4,20 & 1,30 \\
\hline & $\begin{array}{l}\text { Eu sei pelo menos uma linguagem de programação de } \\
\text { computadores }\end{array}$ & 15 & 2,93 & 1,03 & 05 & 3,20 & 1,09 \\
\hline
\end{tabular}

Me: média; DP: desvio padrão. Fonte: Autores.

A resposta sim de 15 entre 21 para a pergunta sobre "sei ao menos uma linguagem de programação" é surpreendente em um país com baixo desempenho em operações básicas de matemática e interpretação de texto. Não fica claro como essa aprendizagem aconteceu (conhecimentos fragmentados em espaços formais como a escola ou projetos sociais, mas também em espaços informais com amigos, etc.); as respostas parecem indicar um aprendizado mais enraizado no cotidiano e no intuitivo informal do que por meio de algum tipo de aprendizagem formal e sistemática.

De acordo com a Tabela 5, 38,10\% dos respondentes consideram o seu nível de conhecimento em programação de computadores como básico, $38,10 \%$ como intermediário e 9,52\% como avançado. 
Tabela 5: Nível de Programação de Computadores.

\begin{tabular}{l|l|l}
\hline Níveis & $\mathrm{N}$ & $\%$ \\
\hline Básico & 08 & 38,10 \\
Intermediário & 08 & 38,10 \\
Avançado & 02 & 9,52 \\
Não respondeu & 03 & 14,29 \\
\hline Total & $\mathbf{1 6}$ & $\mathbf{1 0 0 , 0 0}$ \\
\hline
\end{tabular}

n=frequência absoluta. Fonte: Autores.

O grupo 1 apresenta os menores escores $(2,38$ a 4,00) em relação à literacia digital dos estudantes. Por outro lado, o grupo 2 exibe os maiores escores (3,20 a 4,80), com destaque para "Sei que informações devo e não devo compartilhar" e "Tenho cuidado de tomar meus comentários e comportamentos adequados à situação em que me encontro on-line” (Figura 1). Essas aprendizagens são importantes considerando que a temática segurança na Internet "precisa de maior atenção por parte da comunidade escolar, principalmente pela democratização do acesso à Internet que chega a oito em cada dez crianças e adolescentes" (Farias et al., 2019, p. 246) no Brasil com faixa etária entre 9 e 17 anos.

Figura 1: Dendrograma do instrumento de inclusão digital.

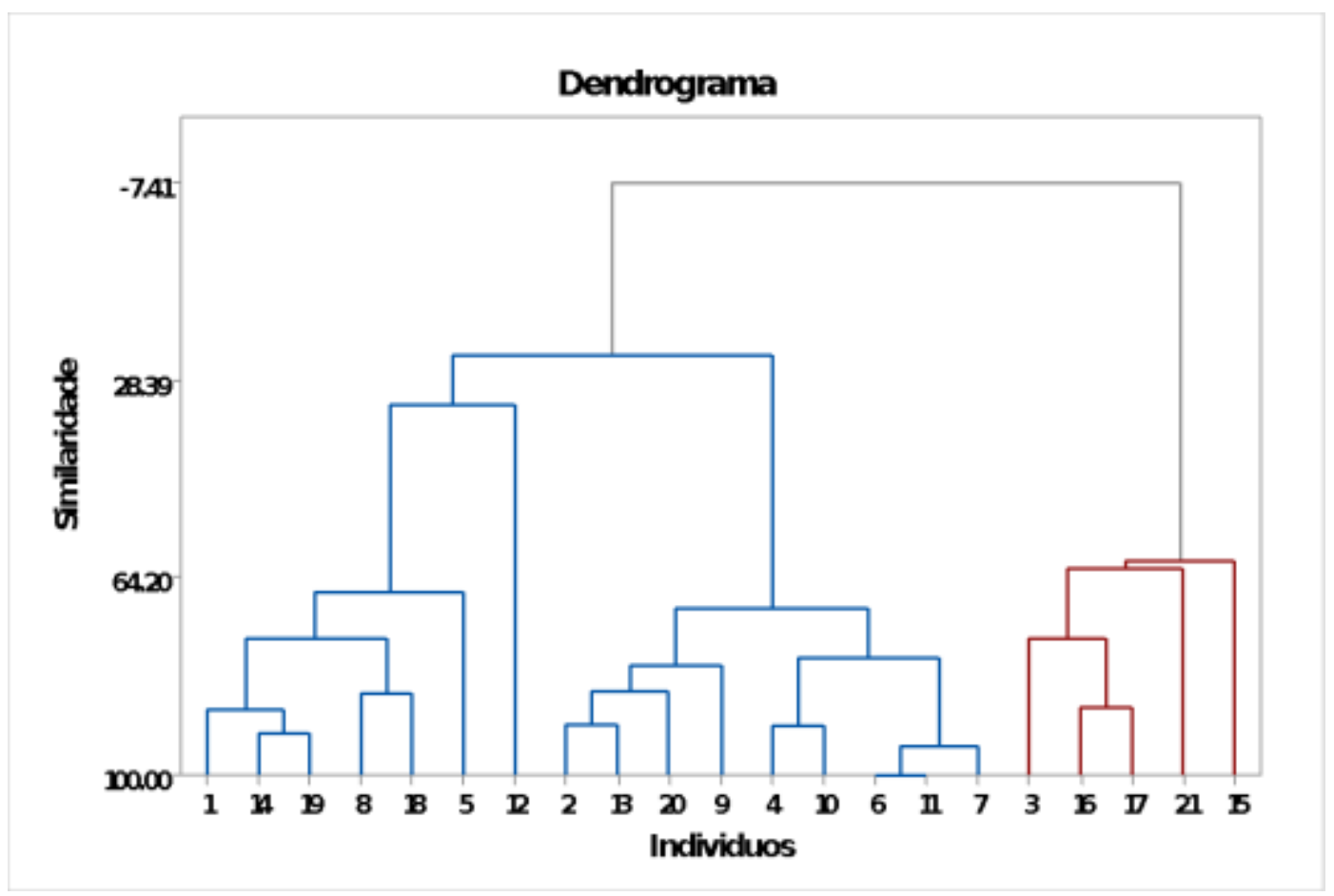

Fonte: Autores.

As perguntas que revelam mais dificuldades para os respondentes são relacionadas a competências que não se adquirem via uso costumeiro de jogos ou de redes sociais (por exemplo, palavras-chaves para pesquisar, encontrar um site que visitou antes). Esse letramento digital se torna cada vez mais importante nos dias atuais, especialmente quando se fala em inserção no mercado de trabalho. De acordo com uma pesquisa realizada nos países membros da OCDE, mais de $95 \%$ dos profissionais que trabalham em grandes empresas e $85 \%$ dos que atuam em empresas de médio porte têm acesso e usam a Internet como parte de seus trabalhos. O índice de acesso e uso nos pequenos empreendimentos é de pelo menos $65 \%$ (OCDE, 2013, p. 46). 


\section{Considerações Finais}

Retomando o que foi posto no início, as variáveis que compõem o tripé da inclusão digital tornam-se mais urgentes do que nunca. A consolidação do uso das tecnologias digitais como elementos de mediação nos processos de ensino e aprendizagem nas políticas de educação do País deve ser enfrentada como pauta central no modelo educacional brasileiro.

Sem o desenvolvimento da literacia digital por parte dos jovens em uma sociedade conectada, o engajamento escolar continuará empobrecido e as chances de empregabilidade diminuirão. Relegar para o terceiro setor o papel de prover inclusão digital, principalmente para as camadas mais vulneráveis da sociedade brasileira, poderá ser entendido como um processo de desresponsabilização do Estado na garantia constitucional de educar.

Como limitação deste estudo destacamos: os resultados apresentados não devem ser generalizados para todos os segmentos juvenis da cidade de Natal (RN), visto que o público estudado foi circunscrito a uma região da cidade com baixa renda salarial. Apesar disso, há indícios para pensar a inclusão digital em zonas periféricas do supracitado município. Para investigações futuras, sugere-se contemplar jovens de diferentes bairros e estratos sociais. No caso do uso de smartphones, há necessidade também de se problematizar o acesso à Internet, ou seja, o que é possível fazer com os pacotes de dados contratados pelos jovens, qual a velocidade da conexão e com quantas pessoas eles compartilham essa franquia de Internet. Indica-se, ainda, investigar como a aprendizagem de linguagens de programação tem ocorrido.

\section{Referências}

Albertin, A. L., \& Albertin, R. M. D. M. (2017). A Internet das coisas irá muito além as coisas. GV-Executivo, 16(2), 12. https://doi.org/10.12660/gvexec.v16n2.2017.68668

Araujo, E. V. F. de, \& Vilaça, M. L. C. (2016). Sociedade Conectada: Tecnologia, Cidadania e Infoinclusão. In Tecnologia, Sociedade e Educação na Era Digital (pp. 17-40). Duque de Caxias, RJ: Universidade UNIGRANRIO. http://blogs.unigranrio.br/informepropep/livro-tecnologia-sociedade-e-educacao-naera-digital-download-gratuito

Comitê Gestor de Internet no Brasil. (2019). Pesquisa sobre o uso da Internet por crianças e adolescentes no Brasil: TIC kids online Brasil 2018. https://www.cgi.br/publicacao/pesquisa-sobre-o-uso-da-internet-por-criancas-e-adolescentes-no-brasil-tic-kids-online-brasil-2018/

Costa, E., Pinto, A., Rodrigues Junior, J., Araújo, A., Pereira, E., Braz Junior, G., Santos, R., \& Rivero, L. (2019). Identificando as Atividades dos Grupos do Programa de Educação Tutorial na Área de Computação no Apoio à Inclusão e Alfabetização Digital. Anais do Workshop de Informática na Escola, 25(1), p. 934. http://dx.doi.org/10.5753/cbie.wie.2019.934.

Farias, F., Medeiros, N., Rocha, S., Medeiros, D., Nóbrega, E., Burlamaqui, A., \& Madeira, C. (2019). Self Protect: Um jogo para auxílio no ensino de conceitos relacionados a Segurança na Internet para Crianças e Adolescentes. Anais do Workshop de Informática na Escola, 25(1), 246. http://dx.doi.org/10.5753/cbie.wie.2019.246.

Gil, Antonio Carlos. (2009). Como elaborar projetos de pesquisa. (4a ed.), Atlas.

Gonçalves, Hortência de Abreu. (2005). Manual de metodologia da pesquisa científica. Avercamp.

Hamelink, C. J. (2001). The Ethics of Cyberspace (SAGE Publi). https://uk.sagepub.com/en-gb/eur/the-ethics-of-cyberspace/book210226.

Hargittai, E., \& Hinnant, A. (2008). Desigualdade digital: diferenças no uso da Internet por jovens adultos. Research Research, 35(5), 602-621. https://doi.org/10.1177/0093650208321782.

IBGE. (2013). Atlas do Censo Demográfico 2010. https://biblioteca.ibge.gov.br/index.php/biblioteca-catalogo?view=detalhes\&id=264529

IBGE. (2018). Pesquisa Nacional por Amostra de Domicílios Contínua - PNAD: Uso de Internet, televisão e celular no Brasil. Diretoria de Pesquisas, Coordenação de Trabalho e Rendimento. https://educa.ibge.gov.br/jovens/materias-especiais/20787-uso-de-internet-televisao-e-celular-no-brasil.html.

IBGE. (2019). Pesquisa de Orçamentos Familiares. https://biblioteca.ibge.gov.br/visualizacao/livros/liv101670.pdf

Lucas, M., \& Moreira, A. (2017). DigComp: Quadro Europeu de Referência para a Competência Digital. https://.doi.org/10.13140/RG.2.1.1606.1049.

Lyotard, J. F. (1988). O inumano: considerações sobre o tempo. Estampa.

Moran, J. M. (2018a). A educação que desejamos: Novos desafios e como chegar lá (5a ed.). Papirus editora. https://books.google.com.br/books?id=PiZe8ahPcD8C\&printsec=frontcover\&hl=pt-BR\#v=onepage\&q\&f=false

Moran, J. M. (2018b). Ensino e aprendizagem inovadores com apoio de tecnologias. In Moran, J. M., Massetto, M. T, \& Behrens, M. A. (orgs.). (2018b). Novas Tecnologias e Mediação Pedagógica (pp. 11-72). Papirus. 
Research, Society and Development, v. 10, n.6, e27310615859, 2021

(CC BY 4.0) | ISSN 2525-3409 | DOI: http://dx.doi.org/10.33448/rsd-v10i6.15859

OECD (2013). OECD Skills Outlook 2013: First Results from the Survey of Adult Skills. OECD Publishing, Paris, https://doi.org/10.1787/9789264204256-en.

Palmeiro, R.; Pereda, V., \& Aires, L. (2017). O papel dos monitores nos espaços de acesso à Internet do Alentejo. In Palmeiro, R.; Pereda, V., \& Aires, L. (2017). Literacia e Inclusão Digital: Boas Práticas em Portugal e em Espanha. Universidade Aberta. Rede ObLID, CEMRI. Lisboa, Portugal.

Parasuraman, A., \& Colby, C. L. (2015). An Updated and Streamlined Technology Readiness Index: TRI 2.0. Journal of Service Research, 18(1), pp. 59-74. DOI: https://doi.org/10.1177/1094670514539730.

Radovanović, D., Holst, C., Belur, S., Srivastava, R., Houngbonon, G., Le Quentrec, E., Miliza, J., Winkler, A., \& Noll, J. (2020). Digital Literacy Key Performance Indicators for Sustainable Development. Social Inclusion, 8(2), pp. 151-167. DOI: http://dx.doi.org/10.17645/si.v8i2.2587

Robin B.R. (2008). Digital Storytelling: A Powerful Technology Tool for the 21st Century Classroom, Theory Into Practice, 47, pp. $220-228$.

Robinson, L. (2018) The identity curation game: digital inequality, identity work, and emotion management, Information, Communication \& Society, 21(5), pp. 661-680, DOI: http://10.1080/1369118X.2017.1411521

Rodrigues, N., \& Batista, M. (2018). Ações e Políticas Públicas de Inclusão Digital: do global ao local, através de conceitos e processos educacionais. Anais do Workshop de Informática na Escola, 24(1), 624. http://dx.doi.org/10.5753/cbie.wie.2018.624.

Silva, J., Soares, R., Garcia, L., Rodrigues, C., Lima, W., \& Silva, A. (2019). A promoção de inclusão digital de estudantes da Educação de Jovens e Adultos (EJA) através da Extensão Universitária. Anais do Workshop de Informática na Escola, 25(1), 227. http://dx.doi.org/10.5753/cbie.wie.2019.227.

Sorj, B. (2003).Brasil@povo.com: a luta contra a desigualdade na sociedade da informação. Jorge Zahar.

Van Deursen, A. J. A. M., Helsper, E., \& Eynon, R. (2016). Development and validation of the Internet Skills Scale (ISS). Information, Communication \& Society, 19, 804-823. https://doi.org/10.1080/1369118X.2015.1078834 